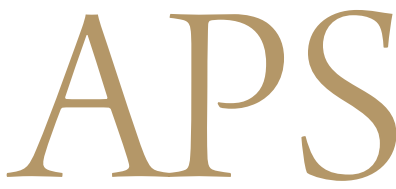

Archives of Plastic Surgery

\title{
Successful local use of heparin calcium for congested fingertip replants
}

\author{
Hideki Kadota ${ }^{1,2}$, Atsushi Imaizumi ${ }^{2}$, Kunihiro Ishida ${ }^{2}$, Yasunori Sashida ${ }^{3}$ \\ ${ }^{I}$ Department of Plastic and Reconstructive Surgery, Kyushu University Hospital, Fukuoka; ${ }^{2}$ Department of Plastic Surgery, Okinawa Chubu \\ Hospital, Okinawa; ${ }^{3}$ Department of Plastic Surgery, Okinawa Hokubu Hospital, Okinawa, Japan
}

Background Conventional methods of external bleeding for congested fingertip replants exhibit notable problems, including uncontrollable bleeding and unpredictable survival of the replant. We have added a local injection of heparin calcium to the routine use of systemic heparinization for inducing external bleeding. We retrospectively examined patients who underwent external bleeding using our method.

Methods Local subcutaneous injections of heparin calcium were made in 15 congested replants in addition to systemic heparinization. Each injection ranged from 500 to 5,000 U. The average duration of the injections was 4.1 days. Surgical outcomes were analyzed and compared with a control group of patients who underwent external bleeding without heparin calcium.

Results The overall survival rate was $93.3 \%$, which was higher than that of the control group $(83.3 \%)$, but the difference was not statistically significant $(P=0.569)$. The survival rate for subzones I and II by the Ishikawa subzone classification was $100 \%$, whereas it was $87.5 \%$ in subzones III and IV. No statistically significant difference was observed. The rate of partial necrosis was $0 \%$ in subzones I and II, whereas it was significantly higher (66.7\%) in subzones III and IV ( $P=0.015)$. The mean total blood loss via external bleeding was $588 \mathrm{~g}$ in 10 fingers. No patients required blood transfusion.

Conclusions Congestion of a replanted fingertip can be successfully managed without blood transfusion by our method. Although complete relief from congestion in replants in subzones I and II is achievable, there is a higher risk of partial necrosis in subzones III and IV.

Keywords Fingertip replantation / Postoperative congestion / External bleeding / Heparin calcium / Chemical leech

\author{
Correspondence: Hideki Kadota \\ Department of Plastic and \\ Reconstructive Surgery, Kyushu \\ University Hospital, 3-1-1 Maidashi, \\ Higashi-ku, Fukuoka, 812-8582, Japan \\ Tel: +81-92-642-5687 \\ Fax: +81-92-642-5687 \\ E-mail: \\ kadohide@qent.med.kyushu-u.ac.jp
}

This article was presented at the 57 th Annual Meeting of the Japanese Society for Surgery of the Hand on April 17-18, 2014, in Okinawa, Japan.

Received: May 27, 2019 • Revised: November 16, 2019 • Accepted: November 23, 2019

pISSN: 2234-6163 • elSSN: 2234-6171 • https://doi.org/10.5999/aps.2019.00815• Arch Plast Surg 2020;47:54-61

\section{INTRODUCTION}

Distal fingertip replantation, which was considered difficult in the past, has recently become a viable choice of treatment due to improvements in surgical techniques and microsurgical equipment that have increased its reported survival rate up to $96 \%$ [1-8]. In distal fingertip amputations, arteries can be iden- tified and repaired in most cases. However, the more distal the level of amputation, the more difficult it becomes to find even a vein for anastomosis [2-5]. In the context of severe tissue damage, such as crush or avulsion injury, difficulties are often encountered when searching for veins to repair.

When replants become congested postoperatively, external bleeding is required to relieve congestion until venous outflow 
is reestablished. Various techniques have been reported to relieve congestion, such as applying medical leeches [6], puncturing or lacerating the replants [9-11], periungual stub incisions or fish-mouth incisions $[2,10,12]$, or making a venocutaneous fistula [13]. These techniques have several drawbacks, such as excessive bleeding, an unpredictable success rate, and the need for frequent monitoring of the replants by experienced medical staff. Although high survival rates have occasionally been reported for external bleeding, blood transfusion is required for most patients [12,14]. An optimal method of external bleeding must fulfill the following requirements: high survival rate of replants, low invasiveness, infrequent interventions, and minimal blood loss [2]. To date, few standardized and ideal protocols have been described for external bleeding of congested replants $[4,10,12,14]$.

Heparin calcium is a highly concentrated heparin preparation that is used as a "chemical leech" to relieve implant congestion [10]. In our facility, induction of external bleeding by local injections of heparin calcium into replants, in addition to routine systemic heparinization, is the method used to manage postoperative congestion of replants. In this study, we investigated the survival rate of replants and the amount of blood loss in patients managed under our protocol for external bleeding.

\section{METHODS}

\section{Patients}

From July 2008 to May 2013, 15 replanted fingertips in 14 patients developed postoperative congestion and were subjected to external bleeding with local injections of heparin calcium in addition to the routine systemic administration of heparin sodium at Okinawa Chubu Hospital (Table 1). There were 13 males and one female, with ages ranging from 6 to 50 years (mean, 31 years). The affected fingers included five index, six middle, and four ring fingers. The level of amputation was categorized according to the Ishikawa subzone classification (Fig. 1) [5,15]. The level was subzone I in two fingers, subzone II in six fingers, subzone III in six fingers, and subzone IV in one finger. All affected fingers were severely damaged by a crush or avulsion injury, and no clean cuts were observed.

\section{Fig. 1. Ishikawa subzone classification}

The level of fingertip amputation is classified into four subzones.

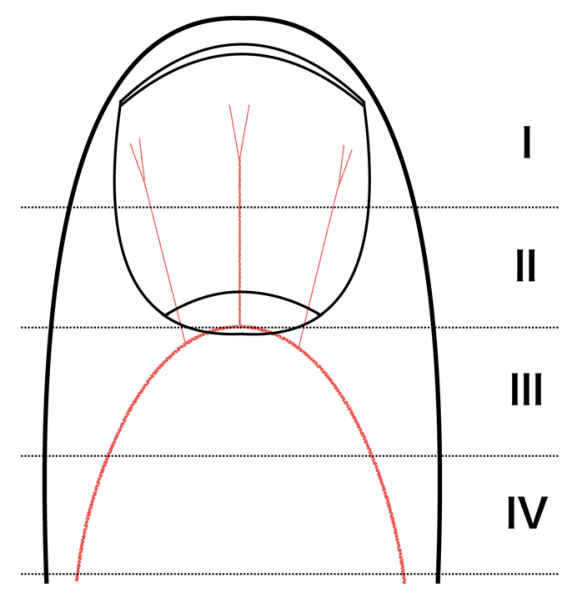

Table 1. Clinical details of congested replants that underwent external bleeding

\begin{tabular}{|c|c|c|c|c|c|c|c|c|c|c|c|c|c|c|}
\hline No. & $\begin{array}{l}\text { Age } \\
(\mathrm{yr})\end{array}$ & Sex & Finger & $\begin{array}{l}\text { Sub } \\
\text { zone }\end{array}$ & Injury & $\begin{array}{c}\text { Arterial } \\
\text { anastomosis }\end{array}$ & $\begin{array}{c}\text { Venous } \\
\text { anastomosis }\end{array}$ & $\begin{array}{c}\text { Minimum } \\
\text { single } \\
\text { dose of } \\
\text { HC (U) }\end{array}$ & $\begin{array}{c}\text { Maximum } \\
\text { single } \\
\text { dose of } \\
\mathrm{HC}(\mathrm{U})\end{array}$ & $\begin{array}{c}\text { Initial } \\
\text { day of } \\
\text { injection } \\
\text { (POD) }\end{array}$ & $\begin{array}{l}\text { Final day } \\
\text { of } \\
\text { injection } \\
\text { (POD) }\end{array}$ & $\begin{array}{c}\text { Maximum } \\
\text { blood loss } \\
\text { (g/day) }\end{array}$ & $\begin{array}{c}\text { Total } \\
\text { blood } \\
\text { loss (g) }\end{array}$ & Outcome \\
\hline 1 & 6 & $M$ & Middle & I & Crush & 1 & None & 500 & 500 & 2 & 2 & 96 & 96 & Survived \\
\hline 2 & 20 & $M$ & Middle & I & Crush & 1 & None & 5,000 & 5,000 & 0 & 0 & 636 & 1,170 & Survived \\
\hline 3 & 23 & $M$ & Ring & $\|$ & Crush & 1 & 2 & 500 & 1,250 & 2 & 4 & - & - & Survived \\
\hline 4 & 38 & $\mathrm{~F}$ & Middle & $\|$ & Crush & 1 & None & 2,500 & 2,500 & 2 & 5 & - & - & Survived \\
\hline 5 & 44 & M & Index & $\|$ & Crush & 1 & 1 & 2,500 & 5,000 & 1 & 6 & 880 & 1,430 & Survived \\
\hline 6 & 10 & M & Index & $\|$ & Crush & 1 & None & 500 & 1,250 & 1 & 3 & 422 & 963 & Survived \\
\hline 7 & 32 & M & Index & $\|$ & Crush & 1 & None & 200 & 500 & 1 & 5 & 255 & 892 & Survived \\
\hline 8 & 36 & M & Middle & $\|$ & Avulsion & 1 & None & 500 & 1,250 & 0 & 5 & 165 & 425 & Survived \\
\hline 9 & 50 & M & Index & |II & Crush & 1 & None & 500 & 2,500 & 0 & 6 & 185 & 265 & Necrosis \\
\hline 10 & 41 & M & Ring & III & Avulsion & 1 & None & 500 & 1,000 & 0 & 6 & - & - & PN \\
\hline 11 & 42 & M & Ring & ||| & Crush & 1 & None & 2,500 & 2,500 & 1 & 1 & 190 & 190 & Survived \\
\hline 12 & 25 & M & Middle & |II & Crush & 1 & None & 1,250 & 1,250 & 3 & 4 & - & - & PN \\
\hline 13 & 30 & M & Middle & III & Crush & 1 & None & 1,250 & 1,250 & 0 & 2 & - & - & Survived \\
\hline 14 & 36 & $\mathrm{M}$ & Ring & ||| & Avulsion & 2 & 1 & 500 & 1,000 & 0 & 5 & 5 & 20 & PN \\
\hline 15 & 36 & M & Index & IV & Avulsion & 1 & None & 500 & 2,500 & 0 & 6 & 165 & 425 & PN \\
\hline
\end{tabular}


One artery was anastomosed in 14 fingers, and two arteries in one finger. One vein was anastomosed in two fingers, two veins in one finger, and venous anastomosis could not be performed in 12 fingers.

\section{Methods}

According to our protocol for fingertip replantation, continuous systemic administration of $12,000 \mathrm{U} /$ day of sodium heparin and $80 \mu \mathrm{g} /$ day of prostaglandin E1 was initiated at the time of arterial anastomosis and continued for 7 days postoperatively to prevent postoperative thrombosis in all patients. Oral administration of $100 \mathrm{mg}$ of aspirin was initiated immediately after surgery and continued for 1 month postoperatively. For pediatric patients, the doses of the medications were reduced based on the instructions of the pediatrician.

When a replanted fingertip exhibited signs of congestion postoperatively, a small fish-mouth incision was made at the most distal part of the replant, and a subcutaneous injection of heparin calcium into the replant was performed once or twice daily. Given the lack of an established protocol for heparin calcium administration, the dose of heparin calcium used in each procedure varied according to the replant and ranged widely from 500 to 5,000 U. Inspection of the color of the replant and blood oozing from the fish-mouth incision helped determine whether the replant remained congested. The dose and dosing interval of heparin calcium injection were titrated according to the complexion of the congested replant. In majority of the cases, the heparin calcium combination treatment was initiated from the day of surgery or on postoperative day (POD) 1 , and the duration of the treatment ranged from 1 to 7 days (mean, 4.1 days).

Surgical outcomes and amount of blood loss were reviewed retrospectively. Whether a replanted fingertip survived or failed was judged by careful daily observation of the color and bleeding from the replant. Even a small area of discoloration suspected of necrosis was diagnosed as partial necrosis. The daily amount of blood loss was determined by measuring the weight of dressings over the replants. In all cases, brisk hemorrhage was induced and continued from the fish-mouth incision using our method of external bleeding. Such bleeding does not occur without arterial anastomosis. Hence, fingertips surviving as composite grafts were not included in this series.

To evaluate the efficacy of using heparin calcium for external bleeding, 12 patients who developed postoperative congestion after fingertip replantation and underwent external bleeding without heparin calcium from 1999 to 2013 were selected as a control group, and their postoperative outcomes were compared with those of patients who received heparin calcium. In the control group, there were 11 males and one female, with ages rang- ing from 2 to 60 years (mean, 37 years). The level of amputation was subzone I for one finger, subzone II for four fingers, and subzone III for seven fingers. One artery was anastomosed in 11 fingers, and two arteries in one finger. One vein was anastomosed in three fingers, and venous anastomosis could not be performed in nine fingers. External bleeding was performed by a fish-mouth incision and/or pin pricking in all patients, with continuous systemic administration of 12,000 U/day of sodium heparin in seven patients.

The Fisher exact test and the unpaired Student t-test were employed for the statistical analyses, and differences exhibiting a Pvalue $\leq 0.05$ were considered statistically significant.

\section{RESULTS}

Fourteen of the 15 replants survived, and the overall survival rate was $93.3 \%$. One case of total necrosis resulted from arterial insufficiency, and was therefore not related to insufficient external bleeding. The overall outcomes were compared with those of the control group. In addition, surgical outcomes and amount of blood loss were compared according to the Ishikawa subzone classification.

\section{Comparison with the control group}

The overall survival rate in the control group was $83.3 \%$. This was lower than that of the heparin calcium group, but the difference was not statistically significant $(P=0.569)$. All two cases of necrosis in the control group resulted from progressive venous congestion due to insufficient external bleeding. The amount of blood loss in the control group was not recorded in the patients' medical charts, but one patient required blood transfusion, assumedly due to an inappropriately large fish-mouth incision and an excessive effect of systemic heparin sodium anticoagulation.

\section{Rate of venous anastomosis}

In the heparin calcium group, venous anastomosis was performed in $25 \%(2 / 8)$ of replants in subzones I and II, compared with $16.7 \%(1 / 6)$ of replants in subzones III and IV. No statistically significant difference was noted $(\mathrm{P}=1.000)$.

\section{Survival rate}

In the heparin calcium group, the survival rate was $100 \%$ for subzones I and II and 87.5\% for subzones III and IV. No statistically significant difference was noted $(\mathrm{P}=1.000)$.

\section{Rate of partial necrosis}

In the heparin calcium group, no partial necrosis was observed in replants in subzones I and II (0\%), while four cases of partial 
necrosis occurred in replants in subzones III and IV (66.7\%), which was a significant difference $(\mathrm{P}=0.015)$.

\section{Blood loss}

The daily amount of blood loss by external bleeding was recorded in the patients' medical charts for 10 fingers in the heparin calcium group (Fig. 2). The total amount of blood loss ranged from $20 \mathrm{~g}$ to $1,430 \mathrm{~g}$ (mean, $588 \mathrm{~g}$ ). In five patients, postoperative hemoglobin levels were measured on POD 1 to 3 . The mean decrease in the hemoglobin level was $4.5 \mathrm{~g} / \mathrm{dL}$ (range, $4.1-5.1 \mathrm{~g} / \mathrm{dL}$ ). No patients required blood transfusion, and oral iron tablets were prescribed for most patients.

The average amount of blood loss of the two fingers in which venous anastomosis was performed at the time of replantation was $725 \mathrm{~g}$, whereas that of the eight fingers without venous anastomosis was $553 \mathrm{~g}$. No significant difference was observed between these two groups $(P=0.342)$.

The mean blood loss was $633 \mathrm{~g}$ in subzone I (two fingers), $795 \mathrm{~g}$ in subzone II (five fingers), $105 \mathrm{~g}$ in subzone III (two fingers), and $425 \mathrm{~g}$ in subzone IV (one finger). The relationship between the total amount of blood loss and the level of amputation was examined by comparing the amount of blood loss in every subzone. However, no statistically significant difference was found.

Two replants experienced a large amount of blood loss, exceeding $1,000 \mathrm{~g}$ in total. In these fingers, the maximum amount of blood loss per day exceeded $600 \mathrm{~g}$, with a single dose of 5,000 U of heparin calcium. In contrast, the minimum amount of total blood loss was $20 \mathrm{~g}$, with a single dose of $500 \mathrm{U}$ of heparin calcium.

All fingers exhibited a reduced tendency to bleed after POD 3.
On POD 7, congestion was relieved in all fingers, and additional external bleeding was no longer required.

\section{Cases}

Case 1

A 6-year-old boy got his middle finger caught in a door, and the finger was completely amputated. The level of amputation was subzone I (Fig. 3). Replantation was performed, and one artery was anastomosed distal to the distal transverse palmar arterial arch. No vein was available for anastomosis. Systemic continuous administration of $2,400 \mathrm{U}$ of heparin sodium daily was performed postoperatively, but congestion of the replant developed and worsened starting on POD 2. A fish-mouth incision was made at the distal edge of the replant, and $500 \mathrm{U}$ of heparin calcium was subcutaneously injected into the replant. Congestion was gradually relieved, and the replant survived without any necrosis. The total amount of blood loss was $96 \mathrm{~g}$.

After 1.5 years, no atrophy was observed, and the patient currently uses his right hand without any limitations.

\section{Case 14}

The ring finger of a 36-year-old man got caught between the hull of a ship and a ladder during scuba diving and was completely amputated. The level of amputation was subzone III (Fig. 4). Replantation was performed, and two arteries and one vein were anastomosed on the volar side. Since congestion of the replant was detected immediately after surgery, a fish-mouth incision was made. Heparin calcium was locally injected once daily (500 U on the day of surgery, $500 \mathrm{U}$ on POD 1, 1,000 U on POD 2, $500 \mathrm{U}$ on POD 4, and $500 \mathrm{U}$ on POD 5). Slow and

\section{Fig. 2. Amount of daily blood loss}

Changes in the amount of daily blood loss due to external bleeding in 10 replants are shown. The red round dotted line shows two cases of excessive bleeding (more than $600 \mathrm{~g}$ per day). The blue linear dotted line shows a case of minimal bleeding (less than $20 \mathrm{~g}$ in total).

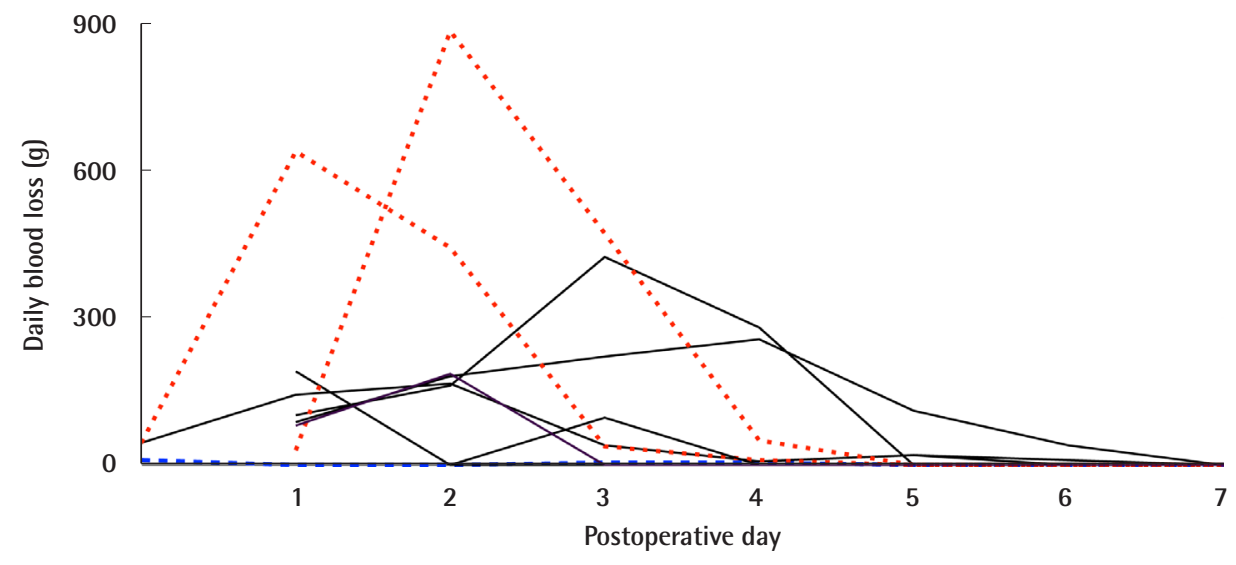




\section{Fig. 3. Replantation in Ishikawa subzone I}

The right middle finger of a 6-year-old boy was amputated by a crush injury. (A, B) A photograph at the initial visit. (C) An anastomosed artery distal to the distal transverse palmar arterial arch. $(D, E)$ Appearance at 1.5 years after surgery. Neither atrophy nor nail deformity was evident (white arrows show the level of amputation.)
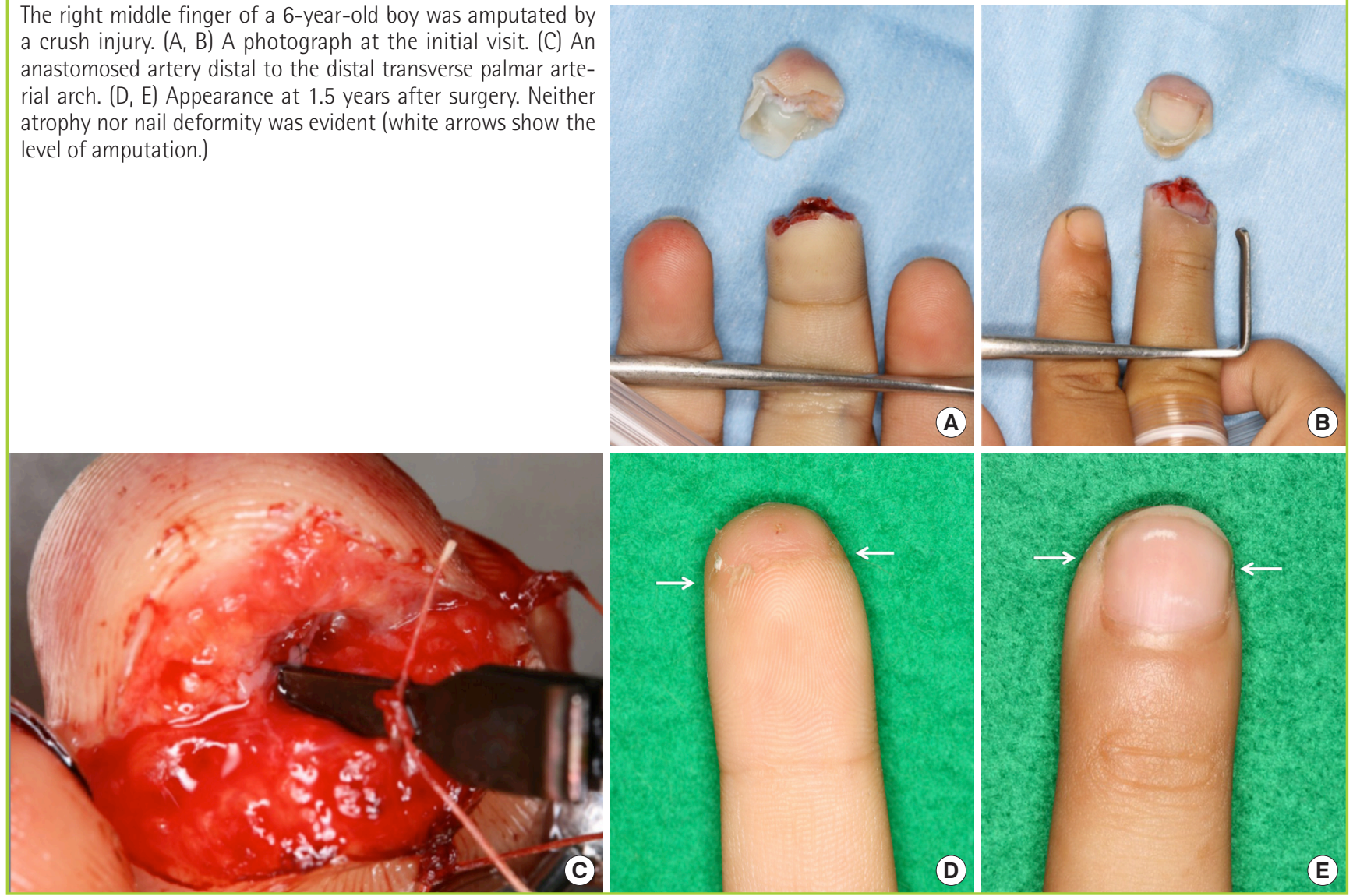

Fig. 4. Replantation in Ishikawa subzone III

The right ring finger of a 36 -year-old man was amputated by an avulsion injury. $(A, B) A$ photograph at the initial visit. (C) Two arteries were anastomosed on the volar side. (D) Partial necrosis occurred at the distal tip of the replant. $\left(E_{1}\right.$ F) Appearance 2 years after surgery. Mild atrophy and nail deformity were evident.
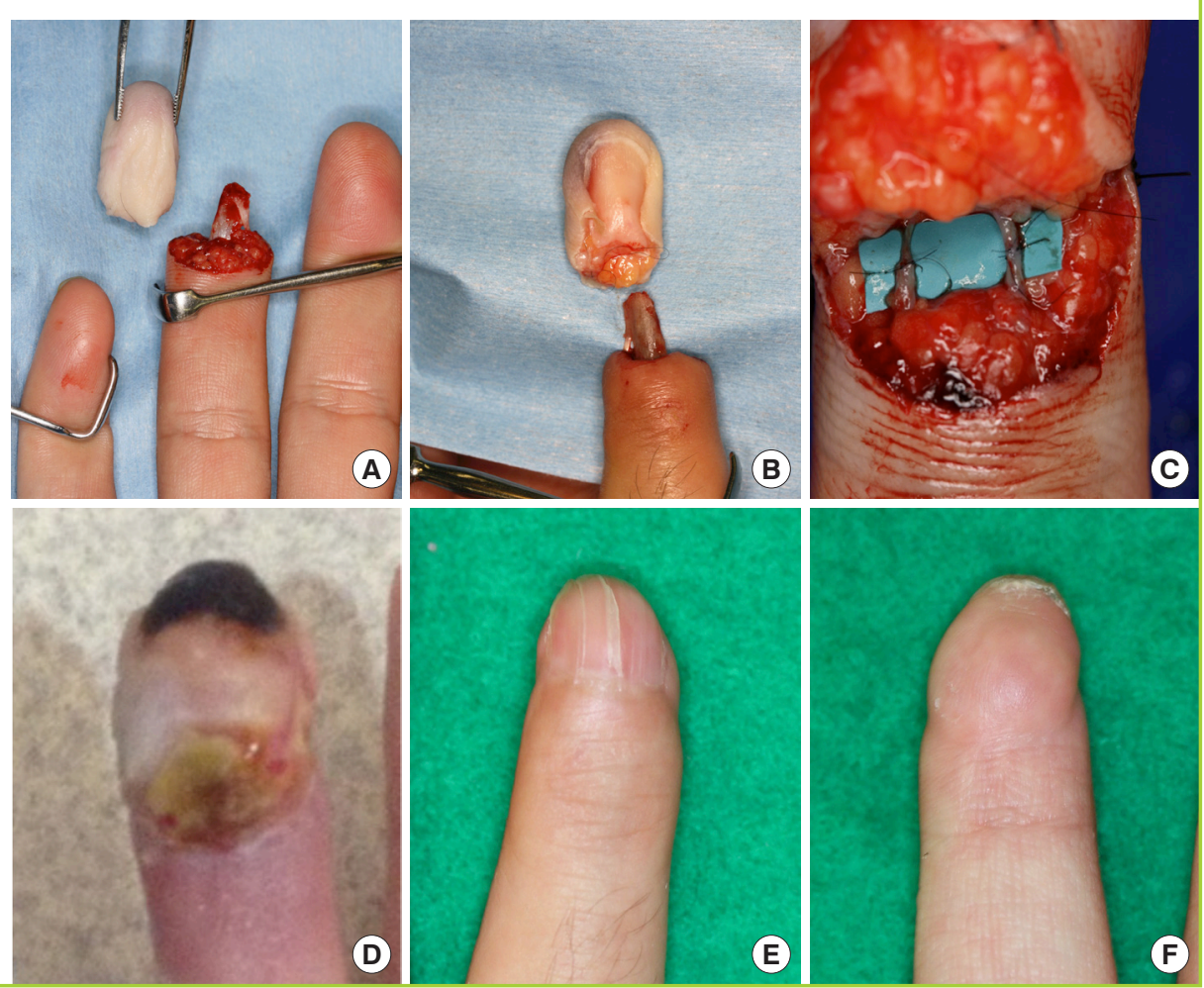
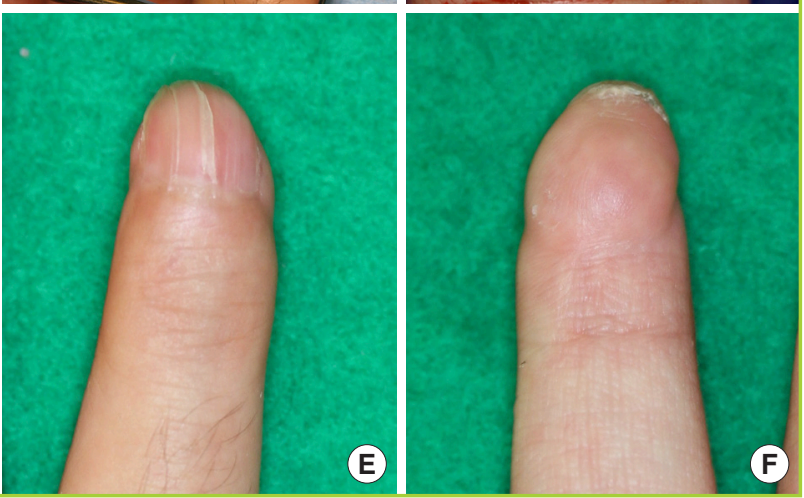
gradual fresh bleeding from the fish-mouth incision was observed (which presents the replant 12 hours after the subcutaneous injection of heparin calcium on POD 2) (Supplemental Video 1). The total amount of blood loss was $20 \mathrm{~g}$ on POD 5. On POD 6, congestion was relieved, and additional external bleeding was no longer required.

After the operation, partial necrosis occurred at the tip of the replant, but all the other parts survived. Mild atrophy occurred 2 years after surgery.

\section{DISCUSSION}

In recent years, high survival rates have been reported for distal fingertip replantation. In a systemic review of 2,273 replanted fingertips, the survival rate of replantations in Ishikawa subzones I and II was reported to be $87 \%$, which is equivalent to that in Ishikawa subzones III and IV [1]. That report implies that even in the most distal fingertip replantations, microsurgical anastomosis is technically possible. However, in our experience, the more distal the level of amputation, the more difficult it is to find a vein to repair, especially in severely damaged fingers [2-5]. When venous anastomosis cannot be performed or thrombosis is present, congestion of the replant is inevitable. If congestion progresses, external bleeding to relieve the congestion is necessary to salvage the replanted fingertip. Ideally, external bleeding should be performed with the least amount of blood loss necessary to maintain a high survival rate of the replantations. However, no previous studies have examined the actual volume of blood loss induced by external bleeding.

Buntic and Brooks [14] reported 100\% survival of 19 arteryonly fingertip replantations in which external bleeding was performed, but $65 \%$ of the patients received blood transfusion. Blood transfusion was also reported to be necessary in up to $88 \%$ of patients undergoing external bleeding in another study [12], and in fact, the need for blood transfusion has been reported in almost all studies. Fingertip replantation is not major surgery; therefore, avoiding blood transfusion whenever possible is ideal.

A characteristic feature of heparin calcium is its higher concentration $(25,000 \mathrm{U} / \mathrm{mL})$ than that of sodium heparin $(10,000$ $\mathrm{U} / \mathrm{mL}$ ). Heparin calcium is long-acting, with a half-life of approximately 3 hours and an inactivation time of approximately 12 hours. Bleeding from a replant can persist for hours with just a single dose of this agent, without the risk of systemic bleeding as it does not readily enter the systemic circulation, especially when the replant becomes congested. A study reported continued bleeding for 4-7 hours after the first subcutaneous injection of $1,000 \mathrm{U}$ of heparin calcium in a replant, and the duration of action was no longer than approximately 6 hours as it was eliminated from the drainage site [10].

At our institution, we combined local injections of heparin calcium with continuous systemic administration of heparin sodium. Few studies have examined the combination of these two types of heparin $[9,12]$, and an optimal protocol has not been established to date. As our protocol was a combination of local and systemic heparin, the frequency of local injections of heparin calcium was once or at most twice daily. During its use, persistent bleeding was observed from the fish-mouth incision, and regular scratching to prevent coagulation of the fish-mouth incision was not necessary. After POD 7, complete relief from congestion was noted in all cases, and additional external bleeding was no longer required. Frequent local injections of heparin calcium are associated with an elevated risk of replant damage [9]. Using our protocol, congestion could be relieved with a low frequency of injections, which were mainly performed once daily without stimulation of the drainage site. This procedure minimized the risk of tissue injury and the burden on both patients and medical staff, providing the greatest advantage of the combined use of local and systemic heparin.

In this study, we achieved a relatively high survival rate of 93.3\%. With the exception of reports with small samples, to the best of our knowledge, our study on external bleeding has reported the highest success rate without blood transfusion. Although the difference was not statistically significant, the survival rate of replants in the patients who received heparin calcium was higher than that of the control group, which contained patients who underwent external bleeding without heparin calcium. In addition, whereas necrosis occurred in two patients in the control group as a result of progressive venous congestion due to insufficient external bleeding, no patients who received heparin calcium experienced insufficient external bleeding. Because of the small number of patients in this study, we could not definitively demonstrate the efficacy of our method with statistical significance. However, we feel that external bleeding using heparin calcium is beneficial for increasing the survival rate and reducing blood loss in clinical practice. A prospective comparative study to confirm the efficacy of our method is needed.

Although we made considerable efforts to minimize blood loss by external bleeding, blood loss exceeding 1,000 g was observed in two fingers. Such a large amount of blood loss is considered excessive. The dose of heparin calcium necessary for external bleeding may vary depending on the volume of the replant and the degree of congestion. Due to the small study population analyzed here, it is difficult to determine the appropriate dose of heparin calcium. A greater number of cases must be evaluated prospectively in the future to provide guidelines for 
the use of heparin calcium. However, we experienced a case where congestion was successfully relieved by local injections of $500 \mathrm{U}$ of heparin calcium once daily with minimal blood loss. This finding suggests that heparin calcium injections could be started with a minimum dose of $500 \mathrm{U}$ to avoid excessive blood loss.

Replantation in subzones I and II was successful in all cases without partial necrosis. These findings suggest that replantations in subzones I and II can survive completely using our protocol for external bleeding. In contrast, replantations in subzones III and IV exhibited a higher rate of partial necrosis than those in subzones I and II. In cases of amputation in subzones III and IV, which involve a larger tissue volume than subzones I and II, external bleeding with heparin calcium could not completely relieve postoperative congestion, which is a limitation of our protocol. For this level of amputation, it may be advisable to consider selecting another method of relieving postoperative congestion, such as delayed venous anastomosis [16]. A further prospective study would be required to determine the appropriate dose of heparin injections and to evaluate the effectiveness of our method of external bleeding.

\section{NOTES}

\section{Conflict of interest}

No potential conflict of interest relevant to this article was reported.

\section{Ethical approval}

The study was approved by the Institutional Review Board of Okinawa Chubu Hospital (IRB No. 2019-54) and performed in accordance with the principles of the Declaration of Helsinki. Written informed consents were obtained.

\section{Patient consent}

The patients provided written informed consent for the publication and the use of their images.

\section{Author contribution}

Conceptualization: Kadota H, Ishida K, Imaizumi A. Data curation: Kadota H. Methodology: Kadota H, Imaizumi A. Project administration: Kadota H, Ishida K. Visualization: Kadota H. Sashida Y. Writing-original draft: Kadota H. Writing-review and editing: all authors. Approval of final manuscript: all authors.

\section{ORCID}

Hideki Kadota https://orcid.org/0000-0001-7833-3524

\section{Supplementary material}

Supplemental Video 1. Slow and gradual fresh bleeding from the fish-mouth incision was seen 12 hours after local injection of heparin calcium on postoperative day 2 .

Supplemental data can be found at: https://doi.org/10.5999/ aps.2019.00815.v001

\section{REFERENCES}

1. Sebastin SJ, Chung KC. A systematic review of the outcomes of replantation of distal digital amputation. Plast Reconstr Surg 2011;128:723-37.

2. Hattori Y, Doi K, Sakamoto S, et al. Fingertip replantation. J Hand Surg Am 2007;32:548-55.

3. Hattori Y, Doi K, Ikeda K, et al. Significance of venous anastomosis in fingertip replantation. Plast Reconstr Surg 2003; 111:1151-8

4. Ito H, Sasaki K, Morioka K, et al. Fingertip amputation salvage on arterial anastomosis alone: an investigation of its limitations. Ann Plast Surg 2010;65:302-5.

5. Ishikawa K, Kawakatsu M, Arata J, et al. Classification of the amputation level of the distal part of the finger: a 10 years study.J Jpn Soc Surg Hand 2001;18:870-4.

6. Scheker LR, Becker GW. Distal finger replantation. J Hand Surg Am 2011;36:521-8.

7. Hasuo T, Nishi G, Tsuchiya D, et al. Fingertip replantations: importance of venous anastomosis and the clinical results. Hand Surg 2009; 14:1-6.

8. Zhang X, Wen S, Wang B, et al. Reconstruction of circulation in the fingertip without vein repair in zone I replantation. J Hand Surg Am 2008;33:1597-601.

9. Tanaka K, Urata S, Arai T. Relief of congestion by subcutaneous injection of Caprocin after replantation of distal phalanx. J Jpn Soc Reconstr Microsurg 2010;23:263-9.

10. Yokoyama T, Hosaka Y, Takagi S. The place of chemical leeching with heparin in digital replantation: subcutaneous calcium heparin for patients not treatable with systemic heparin. Plast Reconstr Surg 2007;119:1284-93.

11. Patradul A, Ngarmukos C, Parkpian V. Distal digital replantations and revascularizations. 237 digits in 192 patients. J Hand Surg Br 1998;23:578-82.

12. Han SK, Lee BI, Kim WK. Topical and systemic anticoagulation in the treatment of absent or compromised venous outflow in replanted fingertips. J Hand Surg Am 2000;25:65967.

13. Kamei K, Sinokawa Y, Kishibe M. The venocutaneous fistula: a new technique for reducing venous congestion in replanted fingertips. Plast Reconstr Surg 1997;99:1771-4. 
14. Buntic RF, Brooks D. Standardized protocol for artery-only fingertip replantation. J Hand Surg Am 2010;35:1491-6.

15. Ishikawa K, Ogawa Y, Soeda $\mathrm{H}$, et al. A new classification of the amputation level for the distal part of the fingers. J Jpn
Soc Reconstr Microsurg 1990;3:54-62.

16. Koshima I, Yamashita S, Sugiyama N, et al. Successful delayed venous drainage in 16 consecutive distal phalangeal replantations. Plast Reconstr Surg 2005;115:149-54. 